Chamorro, J., Marmolejo-Avenia, G. \& Insuasty-Portilla, E. (2019). Una experiencia del recorrido territorial de una etnoeducadora inga. La localización en el desarrollo del pensamiento matemático. Revista

Latinoamericana de Etnomatemática, 12(4), 6-22. DOI: 10.22267/relatem.19124.31

Artículo recibido el 28 de agosto de 2019. Aceptado para publicación el 10 de diciembre de 2019

\title{
Una experiencia del recorrido territorial de una etnoeducadora inga. La localización en el desarrollo del pensamiento matemático
}

\section{A territorial travel experience an etnoeducadora inga. The case of the location in the development of mathematical thinking}

\author{
Julian Chamorro Becerra ${ }^{1}$ \\ Gustavo-Adolfo Marmolejo-Avenia ${ }^{2}$ \\ Edwin-Giovanni Insuasty-Portilla ${ }^{3}$
}

\begin{abstract}
Resumen
Un desafío de la sociedad es convivir con la diversidad cultural, para consolidar procesos educativos que concienticen el valor de la singularidad, la pluralidad y la complementariedad. Sin embargo, la enseñanza de las matemáticas ignora el pensamiento matemático desarrollado en comunidades indígenas como objeto de reflexión, de modo que la caracterización, sistematización y divulgación de cómo las matemáticas son o han sido tratadas en contextos educativos de comunidades indígenas, es una cuestión a considerar. Esta investigación visibiliza elementos culturales y sociales afiliados a actividades pedagógicas que gestiona una etnoeducadora Inga de Santiago (Valle de Sibundoy, Colombia) para el desarrollo de pensamiento matemático. En particular, se contempla el rol de la localización en procesos de espacialidad. El estudio se desarrolló en la Institución Etnoeducativa Rural Bilingüe Iachai Wasi Carlos Tamabioy de Santiago. Se seleccionó el método de investigación cualitativa a fin de obtener una rica comprensión de la visión matemática y su uso para el Inga. Puntualmente, se consideró el Estudio de Caso. Cinco categorías de análisis se consideraron para caracterizar los tipos de localización privilegiados en la investigación: Memoria Histórica, Rito cultural, Cooperativismo, Motivación y Percepción espacial. Fueron identificadas tres formas de referenciar el espacio que destacan la complejidad de la localización en condiciones socio-culturales y su inclusión en ambientes de enseñanza: literal, esquemática, auto-identificación. Los resultados aportan al fortalecimiento de procesos pedagógicos etnomatemáticos y contribuyen a la explicitación de pensamiento matemático propio.
\end{abstract}

Palabras claves: actividades matemáticas universales; localización; espacialidad; etnometodología; recorrido territorial y memoria histórica.

\begin{abstract}
A challenge for society is to live with cultural diversity. This allows the consolidation of educational processes that raise awareness of the value of singularity, plurality and complementarity. But, the teaching of mathematics ignores as a reflection object the mathematical thought developed in indigenous communities. The characterization, systematization and dissemination of how mathematics is or has been treated in educational contexts of indigenous communities, then, is a question to consider. The research makes visible cultural and social elements affiliated to pedagogical activities that an ethnic educator in the Inga indigenous shelter of
\end{abstract}

${ }^{1}$ C.E.M El Cerota. Pasto, Colombia. Contacto: jhchb9837@yahoo.com.co

${ }^{2}$ Universidad de Nariño. Pasto, Colombia. Contacto: usalgamav@ gmail.com

${ }^{3}$ Universidad de Nariño. Pasto, Colombia. Contacto: edwinsuasty@gmail.com 
Santiago (Valle de Sibundoy) manages when developing mathematical thinking. In particular, referred to the role of locating in processes of spatiality. The study was conducted in the institution Etnoeducativa Rural bilingual Iachai Wasi Carlos Tamabioy at Santiago. The qualitative research method has been selected, in order to obtain a rich understanding of the mathematical view and its use for Inga people. Specifically, the Case Study was considered. Five categories of analysis were considered to characterize the types of location privileged in the research: Historical Memory, Cultural Rite, Cooperativism, Motivation and Spatial Perception. Three ways of referencing space were identified: literal, schematic, self-identification. These types of localization highlight the complexity of localization in socio-cultural conditions and its inclusion in teaching environments. The results contribute to the strengthening of ethnomathematical pedagogical processes and contribute to make explicit the Inga mathematical thinking.

Keywords: universal mathematical activities; location; spatiality; ethnomethodology; territorial travel and historical memory.

\section{INTRODUCCIÓN}

En el mundo occidental la matemática tiende a exponerse como una disciplina única, universal y sin relación con la cultura (Bishop, 2005). No obstante, desde hace cuatro décadas, la Etnomatemática ha promovido la valoración del pensamiento matemático de las comunidades indígenas y de otros grupos culturales. Desde este punto de vista, la Etnomatemática constituye una herramienta que proporciona elementos para reivindicar saberes locales, lo cual permite valorar y visibilizar el conocimiento matemático del colonizado frente al conocimiento matemático del colonizador (Blanco-Álvarez \& Oliveras, 2016).

Esta investigación se enmarca en el campo de investigación de la Etnomatemática. Estudia la Localización como una de varias prácticas culturales posibles (Bishop, 1999). Su propósito es identificar los tipos de localización que una etnoeducadora Inga promovió al realizar un recorrido territorial. Para lograr dicho propósito se consideró como pregunta orientadora ¿Cuáles son los tipos de localización que se identifican en el territorio Inga y qué referentes participan en cada uno de ellos?

La revisión bibliográfica realizada en la investigación permite afirmar que son pocos los estudios cuya atención recae en la localización como práctica cultural. Por ejemplo, Lira \& Iglesias (2013) analizaron cómo los albañiles asumen decisiones de ubicación al realizar modificaciones o construcciones en una vivienda. Suárez, Acevedo \& Huertas (2009), por su parte, al considerar como objeto de estudio a la población no vidente, describieron experiencias de lateralidad, ubicación y semejanzas. Otros estudios, como el de Mesquita 
Chamorro, J., Marmolejo-Avenia, G. \& Insuasty-Portilla, E. (2019). Una experiencia del recorrido territorial de una etnoeducadora inga. La localización en el desarrollo del pensamiento matemático. Revista Latinoamericana de Etnomatemática, 12(4), 6-22. DOI: 10.22267/relatem.19124.31

(2004) y Aroca (2012), consideraron, respectivamente, el concepto de espacio en niños de la calle brasileños y las formas de ubicación privilegiadas por grupos de pescadores, antes y durante sus faenas de pesca.

En cuanto a los reportes de investigación que enriquecen y amplían la mirada sobre la espacialidad en contextos indígenas su divulgación aún es más escasa. Entre los pocos detectados destacan el trabajo de Rik Pinxten (1983) y Caicedo et al. (2012). Mientras que en el primer caso se consideró la idea de ubicación y de espacialidad en la comunidad indígena Navajo de los Estados Unidos; en el segundo caso, la atención recayó en la explicitación de elementos conceptuales, prácticos y ejemplificantes para favorecer la transformación curricular, la formación de etnoeducadores y la integración de las familias en los procesos educativos de la cultura Nasa.

\section{MARCO CONCEPTUAL}

Un desafío de la sociedad es convivir con la diversidad cultural para consolidar procesos educativos que concienticen el valor de la singularidad y la pluralidad. Es necesario aceptar diversas formas de pensar y proceder, ninguna superior a otra. En lo educativo, el reconocimiento a la diversidad exige considerar articuladamente aspectos políticos, sociales y culturales. Los cuales desencadenan acciones y exigencias colectivas. Los enfoques socioculturales, pues, desempeñan un rol transcendental en la transformación de tales dinámicas: el desarrollo del individuo se sustenta en las interacciones con los demás y está inmerso un contexto cultural y social, este da forma a su realidad. En ese contexto queremos explicar dos conceptos importantes para abordar este tema desde una perspectiva sociocultural.

\subsection{Etnometodología:}

La Etnometodología posibilita formas de expresión que organizan y describen la experiencia social. Es el caso, de los modelos normativos quienes regulan las conductas de los actores sociales - Cooperativismo y Motivación-; la racionalidad práctica de las actividades cotidianas - Memoria Histórica y Rito cultural- y el tipo de conocimiento social puesto en práctica de forma implícita - percepción espacial-. Elementos asumidos como categorías de análisis en la presente investigación. Así pues, un sujeto, está dotado de un conjunto de 
procedimientos, de métodos, de actividades, los cuales le permiten inventar dispositivos de adaptación para dar sentido al mundo que le rodea.

La Etnometodología alude al sentido y desarrollo de acciones cotidianas (comunicación, toma de decisiones, razonamientos) en forma de razonamiento práctico (Garfinkel, citado en Coulon, 1987). Asume que la auto-organización del mundo social no se sitúa en el estado, la política o cualquier superestructura abstracta. Al contrario, contempla que debe considerarse a través de las actividades prácticas de la vida cotidiana, espacio donde los miembros de la sociedad le asignan forma a partir de los presupuestos y tipos de conocimientos propios del sentido común.

Los modelos normativos reguladores de las conductas de los actores sociales, la racionalidad práctica de las actividades cotidianas y el tipo de conocimiento social implícitamente puesto en práctica, ofrecen procedimientos de interpretación que cada actor emplea para dar sentido a sus acciones y a las de los demás. Suponen además una armazón para la existencia de un mundo significante exterior e independiente de las interacciones sociales, la cual establece un bricolaje o invención permanente de la realidad social. No obstante, este procedimiento tiene un carácter paradójico puesto que trabaja de manera empírica problemas cuya naturaleza había sido considerada como filosófica.

\subsection{La localización:}

Desde la perspectiva sociocultural, las matemáticas son una manera de conocer y son un fenómeno pancultural. En palabras de Bishop (1999), las matemáticas occidentales constituyen una variante particular de la matemática desarrollada a través de los tiempos por diversas sociedades. Su estudio debe asumir la génesis de las ideas matemáticas reconociendo y al mismo tiempo demostrando sus relaciones con la cultura. Un elemento determinante es el simbólico, es decir, todo grupo de "conceptualizaciones explicativas significativas en la tecnología simbólica de las matemáticas" (p. 131).

Existe una serie de actividades las cuales operan como "conceptos organizadores que proporcionan el marco de conocimiento" (Bishop, 1999, p. 132) de las matemáticas: contar, medir, jugar, explicar, localizar, diseñar. Las dos primeras, estructuran lo numérico; el juego induce lo lúdico y la explicación, la ampliación, generación y comunicación de conocimiento; las dos últimas, consideran lo geométrico. 
Chamorro, J., Marmolejo-Avenia, G. \& Insuasty-Portilla, E. (2019). Una experiencia del recorrido territorial de una etnoeducadora inga. La localización en el desarrollo del pensamiento matemático. Revista Latinoamericana de Etnomatemática, 12(4), 6-22. DOI: 10.22267/relatem.19124.31

La caracterización de estas actividades permite identificar similitudes y diferencias entre grupos sociales distintos ya que las formas cómo una cultura y otra acceden a dichas actividades depende del entorno local y de las necesidades provocadas. Por otra parte, el lenguaje y las formas de presentación asociadas varían según la relación de la cultura con el medio. De manera que considerar información sobre lo que una cultura puede hacer, permite aprender sus puntos fuertes y controlar el culturo-centrismo que solemos padecer cuando contemplamos culturas distintas a la nuestra.

La localización, cuestión de interés en la investigación, alude a la necesidad humana básica de "satisfacer la coexistencia entre mente, cuerpo, alma y entorno" (Bishop, 1999, p. 54). "Destaca los aspectos topográficos y cartográficos del entorno" (p. 42). Junto a la actividad de diseño aporta ideas geométricas que permite conceptualizar el espacio. Enfatiza la geometría espacial de la posición y del movimiento controlado. No debe ser un mero ejercicio de lápiz y papel. Los conceptos deben derivarse de actividades en el entorno inmediato y accesible, asimismo, de la codificación y la simbolización de los resultados de esas actividades. En breve, la localización desarrolla "el lenguaje, las imágenes espaciales y los sistemas de coordenadas" (p. 67).

La exploración motivada por conocer el territorio y la búsqueda de alimento relaciona la localización con el desarrollo de pensamiento matemático. De esta forma, las comunidades establecen vínculos con los entes y acciones que habitan su contexto espacial, permiten diseñar métodos para suscitar su codificación y simbolización. Conocer el espacio ${ }^{4}$ donde se vive, saber desplazarse, viajar sin perderse y relacionar objetos entre sí para ubicarse o

\footnotetext{
${ }^{4}$ Pinxten, Van Dooren y Harvey (en Bishop, 1999, p. 49) determina tres niveles de espacio: físico o de objetos, cosmológico y socio-geográfico. Mientras que el primero incluye una percepción sensible de las propiedades que posee el espacio y en el segundo se abarca la lógica asentada en un pensamiento mítico para arreglar u ordenar el espacio geográfico, el tercero considera el intelecto del ser humano para estudiar o describir el medio tal y como la experimenta en la vida cotidiana lo evidencia, es decir, como se ve, siente, palpa, huele, recuerda e imagina dentro de un plano cultural. Nuestra atención recae en el último de ellos, su importancia radica en que suscita no solo nociones geométricas sino también de dirección, orden y finitud (Bishop, 1999) por el cual contribuye a configurar la cardinalidad del número natural y la actividad matemática universal de contar. En lo que sigue, hace parte de una lista de categorías que aportan en el análisis del trabajo: abierto/cerrado; izquierdo/derecho; alto/profundo; absoluto/relativo; lejano/cercano; sobre/bajo; encima/debajo; interno/externo; reposo/movimiento; Finito/infinito; convergente/divergente; vertical, perpendicular (dimensión); anterior/posterior (enfrente de, detrás de).
} 
realizar un mapeo del entorno, son ideas dependientes de la relación e interacción que se establece con el entorno natural y sociocultural.

A manera de ejemplo, en la Tabla 1 se describen algunas nociones básicas que determinan la localización y se reseñan los conceptos matemáticos alusivos.

\begin{tabular}{|l|l|}
\hline \multicolumn{1}{|c|}{ Nociones } & \multicolumn{1}{|c|}{ Conceptos que organiza } \\
\hline - "Todas las sociedades han desarrollado métodos más o menos & - Preposiciones \\
sofisticados para codificar y simbolizar su entorno espacial” (p. 48). & - Descripciones de recorridos \\
- La necesidad relacionada con el entorno estimula & - Localización en el entorno \\
- La manera como se organizan las ideas espaciales, la distinción & - Orientación en la brújula \\
parte/todo y la naturaleza dinámica-estática del espacio, difiere de una & - Ubicaciones en el espacio \\
cultura a otra. & - Viajes (distancia) \\
- el desarrollo de ideas específicas de localización considera el & - Líneas (rectas y curvas) \\
"conocimiento del paisaje en relación con sus mitos e historia dentro de & - Ángulo como giro \\
ese paisaje" (p. 51), & - Rotaciones \\
- Las características del entorno influyen en el lenguaje y la representación & - Sistemas de localización: \\
de localizar la capacidad de orientación & coordenadas polares y 2D/3D \\
- Algunas culturas poseen una brújula interiorizada (sur, norte, este y & - Mapas: Latitud y longitud \\
oeste) que les permite hablar de dicho sistema y su empleo. & - Lugar geométrico \\
- Las relaciones de los sujetos con las estaciones, las direcciones, la & - Vector \\
temperatura y el sol determinan formas distintas de localización. & - Espiral \\
\hline
\end{tabular}

Tabla 1. Nociones que determinan la actividad universal de Localización y conceptos matemáticos que organiza

Fuente: Bishop (1999)

\section{METODOLOGÍA}

Esta es una investigación de naturaleza cualitativa que contempla el estudio de caso para explorar y describir la sinergia social y cultural presente en la actividad matemática universal de localización (Bishop, 1999) a través de un recorrido territorial Inga ${ }^{5}$. La captación, selección y análisis de la información, así como la determinación y manipulación de las variables de análisis, se realizó de manera inductiva: provienen de las circunstancias que provocan las acciones y actuaciones de una etnoeducadora de la Institución Etnoeducativa Rural Bilingüe Iachai Wasi Carlos Tamabioy (Resguardo Indígena Inga de Santiago-

\footnotetext{
${ }^{5}$ Nambikuna wakachidu o caminos de investigación: estrategia de trabajo vinculada a la construcción y actualización de conocimiento colectivo, donde la caracterización de lo que sienten, dialogan, interpretan y la forma cómo actúan los individuos de una comunidad, son cuestiones utilizadas para determinar cuál es su aporte a la constitución de conocimiento matemático en contexto. Donde los Mayores y Mayoras de la comunidad tejen, desentrañan y articulan los saberes, conocimientos, mitos, costumbres determinantes del pueblo Inga; renovando la memoria colectiva y resignificado su cultura. Todos aportan al desarrollo del pensamiento ancestral a través de la construcción de imaginarios colectivos y representaciones simbólicas, con miradas más allá de lo aparente, facilitando los procesos de aprendizaje y construcción de conocimiento.
} 
Chamorro, J., Marmolejo-Avenia, G. \& Insuasty-Portilla, E. (2019). Una experiencia del recorrido territorial de una etnoeducadora inga. La localización en el desarrollo del pensamiento matemático. Revista Latinoamericana de Etnomatemática, 12(4), 6-22. DOI: 10.22267/relatem.19124.31

Putumayo), quien suscita y lidera el recorrido territorial en estudio. La formación profesional de la Etnoeducadora es la Etnolingüística, no obstante, es ella quien, a nivel de la primaria, imparte las clases de todas las asignaturas, entre ellas las de matemáticas.

En cuanto a los instrumentos de recolección de datos utilizados para para acopiar los datos de la investigación se consideraron los expuestos en Bisquerra (1989) para estudios de naturaleza cualitativa, es el caso, de registros de audio y visuales (videos, grabaciones), notas de campo y, en casos puntuales se utilizaron entrevistas semi-estructuradas con el objeto de ampliar o precisar las descripciones y explicaciones analizadas.

Trabajo de campo: La etnoeducadora, en conjunto con una sabedora de la comunidad, organizó la actividad escolar enfocada a una práctica ancestral: el recorrido territorial. El diseño pedagógico se elaboró durante cuatro días en jornada extraescolar y su ejecución consideró una sesión de 6 horas con estudiantes de segundo de secundaria -grado séptimo-. Se enfatizó en el proceder de la localización que participa en la configuración del pensamiento matemático Inga. Los procedimientos privilegiados fueron: reconocer lugares sagrados del pueblo Inga aledaños a la comunidad estudiada; visibilizar un pensamiento mítico asociado a elementos culturales y sociales para realizar una ubicación y visibilizar las estrategias que la sabedora usa para localizar.

El recorrido territorial consideró un trabajo grupal (sabedora-etnoeducadora-estudiantes) seguido de la presentación y puesta en común de las maneras de proceder en grupo, por ejemplo, reglas de comportamiento, actividades a realizar y ofrenda. Por último, los estudiantes consignaron en sus cuadernos de notas las maneras como se ubica/localiza los lugares sagrados y otros seres dentro del territorio Inga del Valle de Sibundoy, para ello se describieron mitos, historias y percepciones espaciales configurados en contexto. La participación de la etnoeducadora se limitó a promover en los estudiantes la realización de descripciones -lo más detallada posible- sobre las actuaciones y maneras de proceder en la localización de la sabedora.

Instrumento metodológico: se contempló la memoria histórica sobre el territorio y las prácticas que le vivifican (y dignifican: ritos culturales) para identificar los elementos que organizan una experiencia social-educativa sobre prácticas ordinarias de una comunidad. Estas hacen parte de una estrategia pedagógica consignada en la propuesta etnoeducativa del 
pueblo Inga de Colombia, las cuales son retomadas por los Inga de Santiago del Valle de Sibundoy. La Mayora y la etnoeducadora son quienes presentan y desarrollan las actividades de una realidad cotidiana donde, de forma maquinal ${ }^{6}$, se dinamizan los conocimientos del mundo bajo la mentalidad del sentido común del actor, imprimiéndose, intersubjetivamente, en numerosas relaciones de interacción que establecen con su territorio y su cultura.

Considerando lo anterior, en el instrumento de análisis se incluyeron cinco categorías. Dos relacionadas con los elementos que organizan una experiencia social-educativa sobre prácticas ordinarias culturales: Memoria Histórica y Rito cultural. Otras dos, asociadas a las interacciones de los actores integradas en modelos normativos que regulan las conductas en la actividad: Cooperativismo y Motivación. Finalmente, se consideró una categoría (percepción espacial), esta alude a las interacciones sensoriales entre los actores y el espacio físico. Las articulaciones de estas cinco categorías de análisis contribuirán a caracterizar el desarrollo de pensamiento matemático promovido en la experiencia analizada en la investigación. A continuación, se definen las categorías y, posteriormente, en la Tabla 2 se presentan y definen los descriptores (formas cómo aparecen las categorías de análisis en el recorrido territorial Inga).

- Memoria Histórica: Recuerdo colectivo, preserva la identidad y continuidad de una comunidad, evoca el valor simbólico-sagrado de las acciones por ella vividas. $\mathrm{Su}$ importancia radica en que la comprensión consumada en las actividades más corrientes de la vida cotidiana: mito, sacralidad del lugar y memoria personal generan sentido (una razón de ser) a las acciones colectivas, las cuales estructuran su mundo (Coulon, 1987). Así, pues, lo familiar, cercano y conocido es tan significativo como aquello que se prefiere evitar, negar y reprimir en el inconsciente. La memoria y la no-memoria son parte de la historia, ambas complementarias e inseparables. Esta categoría, explica, en parte mitológicamente, los acontecimientos y particularidades originales de la historia y la cosmogonía de los pueblos, también los sistemas de símbolos que se establecen en la geografía y en la sacralización de espacios donde se organiza y delimita el mundo natural, social y espiritual.

- Rito Cultural: Práctica cotidiana de la comunidad, configura sus modelos normativos para generar cultura. Las prácticas que los actores (Mayora, etnoeducadora y estudiantes) emplean para asignar sentido a sus acciones marca un carácter social en la estructura de

\footnotetext{
${ }^{6}$ Los conocimientos sobre el mundo son expuestos, tratados y significados de acuerdo al carácter que rutinariamente es asignado en la escena social de donde emergen (Coulon, 1987)
} 
Chamorro, J., Marmolejo-Avenia, G. \& Insuasty-Portilla, E. (2019). Una experiencia del recorrido territorial de una etnoeducadora inga. La localización en el desarrollo del pensamiento matemático. Revista

Latinoamericana de Etnomatemática, 12(4), 6-22. DOI: 10.22267/relatem.19124.31

mundo de cada individuo (Garfinkel, citado por Coulon, 1987). A través de un intercambio de puntos de vista y la conformidad de un sistema de pertinencia se disipan las divergencias de percepción del mundo. La 'actitud natural' encierra la capacidad para tratar a los objetos $\mathrm{y}$, más generalmente, las acciones y los acontecimientos de la vida social con vistas a conservar un mundo en común. Dentro de la comunidad indígena Inga, conservar lo común de sus acciones y los acontecimientos se configura en ritos culturales donde la actividad se presenta como agrado y ofrenda.

- Cooperativismo: Formas de participación de los actores que promueven el desarrollo de la actividad. Los actores interpretan y configuran el mundo según la naturaleza de sus interacciones con él. Los actores se valen de métodos para tomar decisiones, razonar, comunicarse, estos, implícitamente, constituyen la realidad social en un bricolaje permanente. En este estado de invención permanente, se pone al día los métodos empleados, los cuales actualizan las reglas que rigen la comunidad. Dichas inter-acciones son variadas por las circunstancias culturales y sociales de las actuaciones de los actores, conllevan a la constitución de su mundo como una imagen de sí mismo y aporta subjetivamente a la configuración de territorio desde las relaciones que construyen con el entorno.

- Motivación: Razones que promueven interés hacia el desarrollo de la actividad planteada. Las motivaciones son parte de los modelos normativos que regulan las conductas e incluso el pensamiento para dar forma a la estabilidad del orden social (Parsons, citado por Coulon, 1987). La relación entre actor y situación no está basada en los contenidos culturales ni en las reglas, sino que nace a partir de procesos de interpretación, los cuales contribuyen a configurar sus acciones.

- Percepción espacial: Interacciones sensoriales entre los actores y el espacio físico, contribuyen al desarrollo de pensamiento matemático orientado a la topografía. Las ideas matemáticas se estructuran teniendo en cuenta el entorno donde son engendradas (Bishop, 1999). En este sentido, la estructuración de conceptos matemáticos geométricos constituye el medio para organizar los objetos, nutriendo las prácticas matemáticas donde los individuos suscitan al ser consciente de las relaciones que establecen con su mundo (Freudenthal, citado por Bishop, 1999).

\begin{tabular}{|c|c|l|}
\hline CATEGORÍA & DESCRIPTOR & \multicolumn{1}{|c|}{ DEFINICIÓN } \\
\hline $\begin{array}{c}\text { Memoria } \\
\text { Histórica }\end{array}$ & Mito & $\begin{array}{l}\text { Expresión ideológica de Mayora utilizada para transmitir (desde la } \\
\text { oralidad) valores, concepciones, significaciones y arquetipos } \\
\text { cosmogónicos. }\end{array}$ \\
\cline { 2 - 3 } & Sacralidad del lugar & $\begin{array}{l}\text { Símbolo cultural inmerso en la cosmovisión indígena Inga que provee } \\
\text { sentido a las concepciones establecidas en el espacio geográfico, son } \\
\text { ellas quienes regulan las acciones y reacciones de la comunidad. }\end{array}$ \\
\cline { 2 - 4 } & Agrado personal & $\begin{array}{l}\text { Recuerdo directo de la Mayora volcado a una experiencia de vida que } \\
\text { contribuye a configurar el valor simbólico para la memoria histórica } \\
\text { actual. }\end{array}$ \\
\hline Rito cultural & $\begin{array}{l}\text { Reconocimiento (material o no material) a la etnoeducadora y a los } \\
\text { estudiantes por el acompañamiento de la mayora en la actividad } \\
\text { planeada. }\end{array}$ \\
\hline
\end{tabular}




\begin{tabular}{|c|c|l|}
\hline \multirow{3}{*}{ Cooperativismo } & Ofrenda & $\begin{array}{l}\text { Tributo de la etnoeducadora, Mayora y estudiantes para los dueños } \\
\text { espirituales de los lugares sagrados por los cuidados ofrecidos. }\end{array}$ \\
\cline { 2 - 3 } & $\begin{array}{c}\text { Nulo } \\
\text { Etnoeducadora }\end{array}$ & $\begin{array}{l}\text { Restricción de la participación de la Mayora y estudiantes en el } \\
\text { intercambio de ideas para el desarrollo de la actividad planeada. }\end{array}$ \\
\cline { 2 - 3 } & $\begin{array}{l}\text { Etnoeducadora- } \\
\text { estudiante } \\
\text { construcción de significados históricos como parte del fortalecimiento } \\
\text { de la identidad cultural. }\end{array}$ & $\begin{array}{l}\text { Apropiación de una imagen de sí mismo por parte del estudiante que } \\
\text { propicia la etnoeducadora. }\end{array}$ \\
\hline Motivación & $\begin{array}{c}\text { Realización personal } \\
\text { espacial }\end{array}$ & $\begin{array}{l}\text { Estimulación a una ocupación profesional por la satisfacción de } \\
\text { realizarla. Lo anterior se desarrolla durante el recorrido territorial en el } \\
\text { que participa la etnoeducadora y estudiante }\end{array}$ \\
\cline { 2 - 4 } & $\begin{array}{c}\text { Desarrollo de } \\
\text { conocimientos } \\
\text { familiares }\end{array}$ & $\begin{array}{l}\text { Conocimientos que, de generación en generación, se han transmitido } \\
\text { entre individuos de una misma familia. Lo anterior acontece en uno de } \\
\text { los puntos de concentración que da lugar en el recorrido para recabar } \\
\text { información sobre plantas medicinales. }\end{array}$ \\
\hline Percepción & Esquemática & $\begin{array}{l}\text { Reside en las formas como los actores articulan los objetos (lugares } \\
\text { sagrados, petroglifos, grupos familiares, entre otros) y las relaciones que } \\
\text { desatan dentro de su espacio para estructurar significados del territorio. }\end{array}$ \\
\cline { 2 - 3 } & Literal & $\begin{array}{l}\text { Formas de articulación a través de las cuales los actores participan y } \\
\text { proveen información acerca de un espacio específico, aportando a la } \\
\text { construcción de identidad geográfica. }\end{array}$ \\
\hline
\end{tabular}

Tabla 2. Descriptores de las categorías de análisis

Fuente: Autores

\section{HALLAZGOS: TIPOS DE LOCALIZACIÓN INGA}

La investigación identificó variadas maneras de referenciar el espacio (literal, esquemática, auto-identificación) que destacan la complejidad de la localización en condiciones socioculturales y su inclusión en ambientes de enseñanza. Se presentan en la Tabla 3 las localizaciones reseñadas, así como los elementos del instrumento metodológico que las determinan.

\begin{tabular}{|l|c|c|c|c|c|}
\hline \multirow{2}{*}{ LOCALIZACIÓN } & \multicolumn{5}{|c|}{ CATEGORÍAS DE ANALISIS } \\
\cline { 2 - 6 } & Cooperativismo & Motivación & $\begin{array}{c}\text { Rito } \\
\text { Cultural }\end{array}$ & $\begin{array}{c}\text { Percepción } \\
\text { Espacial }\end{array}$ & $\begin{array}{c}\text { Memoria } \\
\text { Histórica }\end{array}$ \\
\hline Literal & $\mathrm{N}$ & $\mathrm{Dcf}$ & $\mathrm{Ag} / \mathrm{Of}$ & $\mathrm{Pl}$ & $\mathrm{Mp} / \mathrm{M} / \mathrm{Sl}$ \\
\hline Esquemática & My-Etn/Etn-Est & Dcf/Rp & $\mathrm{Ag} / \mathrm{Of}$ & $\mathrm{Pe}$ & $\mathrm{Mp} / \mathrm{M} / \mathrm{Sl}$ \\
\hline Auto-identificación & Etn-Est/My-Etn & Dcf/Rp & $\mathrm{Ag} / \mathrm{Of}$ & $\mathrm{Pl} / \mathrm{Pe}$ & $\mathrm{Sl} / \mathrm{M}$ \\
\hline
\end{tabular}

Tabla 3. Localizaciones promovidas en el Recorrido territorial Inga. Elementos de caracterización. Fuente: Autores.

En lo que sigue se describirán los tipos de localización identificados y los comportamientos e importancia que suscitan. La atención recae en cómo los actores recurren a las localizaciones reseñadas y cómo articularon la percepción espacial al referenciar su ubicación 
Chamorro, J., Marmolejo-Avenia, G. \& Insuasty-Portilla, E. (2019). Una experiencia del recorrido territorial de una etnoeducadora inga. La localización en el desarrollo del pensamiento matemático. Revista Latinoamericana de Etnomatemática, 12(4), 6-22. DOI: 10.22267/relatem.19124.31

a partir de sus conocimientos sobre sus círculos familiares y socio-culturales. En algunos casos, a modo de ejemplo, se presentarán fragmentos de los registros realizados.

Localización literal: considerado al socializar "deductivamente" conceptos espaciales (formas, superficies y bordes) incluidos en el recorrido territorial.

Para mantener vivos los ritos culturales como prácticas cotidianas que se transmite de una generación a otra y establecer la organización y ordenamiento territorial, la comunidad Inga caracteriza los medios naturales (lugares sagrados, petroglifos, entre otros) por sus formas, contornos, espacios, etc. Asimismo, resalta las relaciones de correspondencia que se construyen con la mitología y la acumulación de experiencias con manifestaciones cosmovisionales. Se genera, pues, un arquetipo de localización cuyas características topográficas y toponímicas del lugar poseen relaciones entre la memoria histórica (mito), la sacralidad de lugar, la cosmovisión y la oralidad.

La participación pasiva de la etnoeducadora y los estudiantes es una característica a considerar en la invención permanente de una realidad social y cultural. Es la Mayora quien suscita el proceso socializador de la tradición cultural tomando en cuenta el conocimiento oral topográfico, toponímico y de cosmovisión suscitado de generación en generación para la sistematización referencial del espacio socio-geográfico.

En ocasiones, los acontecimientos históricos y míticos consignados en algunos lugares, carecen de significado para los estudiantes y la etnoeducadora quienes no los asumen como referencias culturales para la localización del lugar. Por ejemplo, la Mayora se detuvo en lugares específicos del trayecto donde se desarrolló el recorrido territorial para narrar hechos históricos y míticos asociados a ellos:

... en este punto de hierbabuena, lo que dejaron en esta cocha que con el tiempo fue creciendo, es la pareja de enamorados que andaban amancebados; que vendrían de por allá bajo. Y, pues, ya no podían convivir juntos porque cada uno ya tenía su pareja por aparte. Ellos querían vivir juntos pero la gente no los dejaba. Entonces, ellos empezaron a andar y andar, pero como ya todos sabían, entonces ellos empezaban hacer el amor sobre la totuma con agua, y con el movimiento ellos iban botando. Eso era el secreto. Entonces, eso es lo que estaban haciendo aquí, pero como los mayores ya sabían, se dieron cuenta y empezaron a gritar a las mamas a una reunión. Ellas se unieron y les cerraron, hasta ahí tuvieron. Ellos tuvieron que levantar y así, esta cocha y otra que 
existen por en el valle es por eso. Que ellos se metían en uno y en otra parte para hacer el amor y afín no los dejaron. De ahí, se fueron al Encano como nadie sabían lo que eran entonces se quedaron viviendo juntos. Quedando encantados los dos. Ese es el origen de la cocha.

Lo anterior, no fue considerado ni por los estudiantes ni la etnoeducadora para caracterizar el lugar en cuestión. Es decir, su percepción espacial no se articuló a aspectos culturales (Mito, Memoria Histórica, la sacralidad del lugar). Al contrario, se contemplaron elementos con características colaborativas y vivenciales que caracterizaron su sentido de orientación.

Localización esquemática: Se identifica en el recorrido hacia la Ciénega cuando se recrea intuitivamente la percepción espacial desde la experiencia colectiva y cooperativa en torno al: a) conocimiento (familiar) y uso de las plantas medicinales, b) las problemáticas sociales que acontecen en la Ciénega, y c) la experiencia de vida de los actores. En este sentido, se aporta a un re-significado del contexto donde se enmarca el mito, la ritualidad y la sacralidad de la Ciénega; en consecuencia, se re-significa el contexto de la memoria histórica de la comunidad, posibilitando la elaboración de un sistema de referencias (que consideran el contexto) para la localización.

Una cuestión fundamental que caracteriza esta localización se relaciona con el proceso de percibir el espacio a partir de referentes determinados por la experiencia directa. Los actores, al articular los objetos existentes y sus relaciones con el espacio-tiempo, pretenden representar el entorno lo más fiel posible a los significados construidos. Por ello, el contexto donde se desenvuelve el mito, la ritualidad y la sacralidad del lugar se tornan en puntos acumulativos de una experiencia, la cual debe re-significarse constantemente según los cambios físicos que de una época a otra transforman geográficamente la Ciénega, a fin de no dejar de lado referentes que permitan considerar conocimientos "reposados" en la memoria colectiva de la comunidad.

Además, el contexto que envuelve el mito, la ritualidad y la sacralidad del lugar (en proceso de re-significación) culturalmente está definido en un sistema de orientación direccional que incluye tanto los movimientos del Sol y de las estrellas como el del planeta Venus. Son ellos quienes reflejan las relaciones de complementariedad imbuidas en la cosmovisión Inga y por tanto son aprovechados por los actores para representar topográficamente su espaciosignificado. Es el caso del procedimiento evidenciado en el siguiente fragmento: 
Chamorro, J., Marmolejo-Avenia, G. \& Insuasty-Portilla, E. (2019). Una experiencia del recorrido territorial de una etnoeducadora inga. La localización en el desarrollo del pensamiento matemático. Revista

Latinoamericana de Etnomatemática, 12(4), 6-22. DOI: 10.22267/relatem.19124.31

Etnoeducador: A ver hijitos, todo lo que nos ha contado la Tía hasta el momento lo deben presentar como ustedes quieran ya sea por escrito o de otra forma. Pero para recordar lo que hicimos. A ver, qué nos ha contado, no se queden callados.

Yeison: $\quad$ Profe, mucho. Habló de plantas medicinales.

Carlos: $\quad$ De los que estaban haciendo el amor y que hicieron esta cocha. Ja ja ja

Ana: $\quad$ Profe, ella sabe mucho de este lugar. Cómo escribimos todo eso.

Etnoeducadora: Pues, solo escriban lo que les llamó mucho la atención.

Ana:

Así, sí sí.

Yeison:

Miren lo que hice del recorrido, pues coloqué las plantas que conocía en este mapa, y el de los amancebados le coloque por acá, para presentarle a la profe.

Carlos: A ver. Todo eso. ¿Ana, donde se cayó Gabriela y que se embarró toditico?

Ana: Eso fue por... recuerdas los ojitos de agua que la Tía nos contaba, por ahí.

Yeison: Eso es por aquí. (Señalando en su mapa).

Carlos:

No. Yo recuerdo que antes de hablar de la planta de salga el mal y entre el bien. Jajaja.

Ana: Pues no sé, ponelo en cualquier parte y ya.

Carlos: No, Yeison ponémolos aquí. Fue aquí.

Yeison: Pero para qué.

Carlos: Pues la profe dijo que coloquemos lo que llamó la atención.

Ana:

Carlos: Pero eso era para lo que decía la Tía.

Mayora: Pero eso me llamo la atención.

Carlos: A ver chiquillos, qué pasa.

Mayora:

Tía podemos colocar en el mapa lo que nos gusta, para presentar a los demás, verdad.

Yeison:

Pues sí. Y qué están haciendo.

Mayora: Estamos haciendo un mapa para presentarle a la profe sobre el recorrido.

Yeison: A ver. ¿Dónde estamos?

Mayora: Aquí Tía.

Yeison: ¿Mmmm, y donde sale taita Sol?

Ana: El Sol sale... Ana ayuda

El Sol sale por acá, pues miren si estamos aquí y donde se cayó Gabriela es aquí, el sol sale por acá.

Mayora: A ver. Mmmm. Por allá sale el taitica. ¿Dónde se cayó Gabriela?

(Est-S): Por allá.

Mayora: Entonces, en la hoja el sol sale por acá y va caminando por acá.

Yeison:

Ana: A ver, claro es por acá.

Yeison: No.

Sí es, mira si Gaby se cayó por allá, en el mapa es aquí. Pues el sol está por acá.

Ana: Verdad. Es que estaba mirando al revés. 
Localización auto-identificación: La codificación del entorno en puntos referenciales a través de características provenientes de la experiencia directa del recorrido territorial o de los conceptos socio-culturales que demarcan una cultura, establecen organizaciones psicológicas que suscitan la percepción de sí mismo como un elemento en un mundo de objetos y sujetos distintos: el ser humano además de los puntos referenciales anclados en un mundo objetivo hace uso de sí mismo como un punto de referencia. Así pues, la percepción del mundo tiene un aspecto anverso: percibirse a sí mismo. No se debe olvidar que la autoconciencia, como un atributo psicológico universal del hombre, es dada al nacer, a través de un esquema tradicional de los puntos de referencia o de un vocabulario de referencia espacial.

Para estar orientado espacialmente en el sentido más amplio (más allá del campo de la percepción inmediata) el individuo, además de ser consciente de sí mismo, debe considerar su posición en algún esquema espacial. Al mismo tiempo, debe mantener la conciencia de sus propios cambios en la situación y ser capaz de asumir la posición de los demás en el esquema con referencia a sí mismo.

El individuo no solo avanza desde un nivel rudimentario a uno más complejo de orientación espacial y movilidad, considera además diversos medios simbólicos para orientarse en un mundo espacial que trasciende su experiencia personal (lugar sagrado, nombre de estrellas, mitos, memorias históricas de colectividades y ritualidades). Todos facilitan en la construcción y mantenimiento de patrones espaciales del mundo donde se vive y actúa.

\section{CONCLUSIONES}

Desde la práctica cultural y social de localizar del pueblo Inga se analizó cómo sus actores promueven el desarrollo de pensamiento matemático propio. Se consideró la percepción espacial y su dinamización con aspectos culturales (sacralidad del lugar, rituales culturales) y sociales (desarrollo de conocimientos en el círculo familiar y formas de cooperar). Asimismo, se evidenció que la localización promueve la movilización de significados culturales y sociales relacionados con prácticas que configuran la racionalización y suscitan el desarrollo de pensamiento entorno a lo espacial y la orientación.

La descripción de los datos arrojó una serie de resultados cuya interpretación desvela algunas conclusiones que merecen ser destacadas: 
Chamorro, J., Marmolejo-Avenia, G. \& Insuasty-Portilla, E. (2019). Una experiencia del recorrido territorial de una etnoeducadora inga. La localización en el desarrollo del pensamiento matemático. Revista Latinoamericana de Etnomatemática, 12(4), 6-22. DOI: 10.22267/relatem.19124.31

La percepción espacial y los conocimientos establecen una plataforma para fijar una orientación espacial: El análisis de las relaciones entre los elementos que caracterizan la localización evidencia que la percepción espacial y los conocimientos familiares establecen un plano o una plataforma, donde los actores suscitan relaciones que fijan puntos de referencia para la orientación espacial. Estos son susceptibles de cambios según los significados provenientes de la experiencia directa y la socialización de esquemas o sistemas de referencia de los actores. Así, pues, la racionalidad con quien se establece dichas relaciones caracteriza un pensamiento cuya visión cosmogónica (desde donde hacen explícita las explicaciones de contexto como parte de la realidad y de la vida) establece una lógica, la cual sustenta la existencia del espacio y el territorio sagrado dentro de la dinámica de la diversidad.

Un espacio sagrado configurado por códigos y lenguajes que determinan una distinción sociocultural del grupo: Destaca un territorio del medio cósmico que reviste de signos, códigos y lenguajes, los cuales indican la sacralidad del lugar y contribuyen a la orientación espacial, teniendo presente las formas y conductas de los actores inspiradas hacia tales espacios. Estas revelan dinámicas y procesos de comunicación articulados con otros espacios sagrados que proveen la subsistencia como grupo socialmente identificable.

Se ilustra así, la aparición del orden y la organización donde aparecen las estructuras, la formas y las normas sociales y culturales que definen fronteras, cuyo principio fundamental rige al pueblo Inga a través de la ley primera (ley de origen, ley madre, ley ancestral), la cual incide en la organización del espacio y el territorio.

La aparición de los sitios sagrados vislumbra la ruptura de la homogeneidad del espacio que permite el tránsito de una región cósmica a otra, a través de procesos propios de comunicación establecidos por los humanos con el medio natural que los rodea. Así las montañas, los árboles, las piedras, el agua, y los caminos se convierten en símbolos y lenguajes, los cuales expresan el vínculo entre el mundo de los espíritus y mundo terrenal. Se trata de signos y códigos que integran el territorio como unidad espacial mítica y real, estableciendo una relación entre el lenguaje espacial y la realidad del lugar que genera el reconocimiento del espacio en toda dimensión. 
Los mitos como conceptualizaciones que estructuran un espacio y su sacralidad: permanecen en la memoria tradicional. Explican los acontecimientos y particularidades originales de la historia y la cosmogonía donde se organiza y delimita el mundo natural, social y espiritual. Así, el mito se acerca a la conceptualización de los sistemas simbólicos que representan las concepciones de las formas reales establecidas en la geografía, evidenciadas en la sacralización de espacios específicos (lagunas, montañas, piedras, entre otras), lo cual conforman la estructura de espacio y territorio sagrado. Estos mantienen relaciones para mantener el equilibrio y regular tanto las acciones como las reacciones de los actores en la diversidad.

El espacio Inga entre la delimitación espacial ancestral y simbólica: Corresponde a la delimitación ancestral y simbólica que representa el territorio sagrado y las dinámicas propias de significar al universo de manera física y real. Los símbolos representan los dominios ancestrales, los seres espirituales (los cuales interactúan con el universo) y los humanos que se manifiestan en los espacios geográficos sagrados, entre otros, montañas, valles, lagunas, piedras, ciénagas. Estos orientan los procesos de supervivencia. Su permanencia depende de la existencia de los distintos seres. Dichas relaciones simbólicas configuran la ritualidad como un proceso espiritual de "pagar" por los beneficios recibidos, estos se manifiestan en los mitos y costumbres trasmitidos de generación en generación, expresan la razón de ser y existir de los pueblos indígenas, así como el de sus territorios.

Finalmente, en cuanto a las contribuciones para el trabajo de un etnomatemático, el tratamiento de la localización dentro de un contexto cultural evidencia que debe incluirse la ubicación y la orientación de los elementos utilizados para promover una representación plana de los entornos visibles conocidos, haciéndose uso de una simbología caligráfica, la cual acude de la experiencia directa (a la vez la caracteriza) y a los elementos culturales reconocidos a través de referencias locales. Dicha representación no recurre a términos cartesianos para considerar las relaciones entre el individuo, el contexto sociocultural y los conocimientos y saberes que componen la cultura. Al contrario, se dinamizan para plantear formas y maneras para organizar y ordenar el espacio según su entorno físico y las bases cosmovisionales del pueblo, en el proceso se generano sus propios términos, y lenguaje para representar. 
Chamorro, J., Marmolejo-Avenia, G. \& Insuasty-Portilla, E. (2019). Una experiencia del recorrido territorial de una etnoeducadora inga. La localización en el desarrollo del pensamiento matemático. Revista

Latinoamericana de Etnomatemática, 12(4), 6-22. DOI: 10.22267/relatem.19124.31

\section{REFERENCIAS}

Aroca, A. (2012). Las formas de orientación espacial de los pescadores de Buenaventura, Colombia. Revista U.D.C.A Actualidad \& Divulgación Científica, 15(2), 457-465.

Bishop, A. (1999). Enculturación Matemática. Buenos Aires: Paidos.

Bishop, A. (2005). Las matemáticas occidentales: el arma secreta del imperialismo cultural. In Aproximación sociocultural hacia la educación matemática (pp. 27-41). Cali, Colombia: Instituto de Educación y Pedagogía, Universidad del Valle.

Bisquerra, R. (1989). Métodos de investigación educativa. Barcelona: CEAC.

Blanco-Álvarez, H., \& Oliveras, M. L. (2016). Ethnomathematics: A political tool for Latin America. RIPEM-International Journal for Research in Mathematics Education, 6(1), 112-126.

Caicedo, N., Guegia, G., Parra, A., Guegia, A., Guegia, C., Calambas, L., \& Diaz, E. (2012). Matemáticas en el mundo Nasa (N. Caicedo \& A. Parra Eds. 2nd ed.). Editorial Fuego Azul: Bogotá, Colombia: CIIIT

Coulon, A. (1987). La Etnometodología. Madrid: Ediciones Cátedra.

Lira, R., \& Iglesias. M. (2013). Casas de Bahareque: Una visión etnomatemática a partir de su construcción. En A, González, J. Sanoja de Ramírez, R. García y Z. Paredes (Edits.), Memorias de VII Jornada de Investigación del Departamento de Matemática y VI Jornada de Investigación en Educación Matemática (pp. 151-162). Maracay: Universidad Pedagógica Experimental Libertador, Instituto Pedagógico de Maracay, Venezuela.

Mesquita, M. (2004). O conceito de espaco na cultural de criança em situatição de rua: Um estudo etnomatemático. En J. P. M. Ribeiro, M. do C. S. Domite y R. Ferreira, R. (Edits.), Etnomatemática: Papel, valor e significado (pp. 125 -136). São Paolo: Zouk.

Pinxten, R. (1983). Anthropology of Space: Explorations Into the Natural Philosophy and Semantics of the Navajo. Philadelphia: University of Pennsylvania Press.

Suárez, I., Acevedo, M., \& Huertas, C. (2009). Etnomatemática, Educación Matemática e Invidencia. Revista Latinoamericana de Etnomatemática, 2(2), 18-51. 